\title{
Avaliação do halotano como agente estressor em frangos
}

\section{Evaluation of halothane as stressor agent in poultry}

\author{
Denis Fabrício Marchi ${ }^{1}$; Alexandre $\mathrm{Oba}^{2}$; Gleice Rocha dos Santos ${ }^{3}$; \\ Adriana Lourenço Soares ${ }^{4}$; Massami Shimokomaki*5
}

\section{Resumo}

Resultados vêm mostrando uma direta relação entre a sensibilidade ao halotano e a ocorrência de filés PSE em aves, que pode ser originado pela influência de fatores ambientais, em que a temperatura é o principal fator. Assim, o objetivo do trabalho foi avaliar o halotano como agente estressor em frangos concomitantemente ao estresse térmico. Neste experimento, 24 frangos de linhagem comercial foram divididos em 6 animais para 4 tratamentos: $\mathrm{HHH}$, frangos submetidos ao teste do halotano e abatidos $1 \mathrm{~h}$ após o teste; HET, frangos submetidos ao teste do halotano e, $48 \mathrm{~h}$ depois, ao estresse de $35^{\circ} \mathrm{C}$ por $1 \mathrm{~h}$, sendo abatidos em seguida; EET, frangos submetidos ao estresse térmico e abatidos imediatamente após este tratamento; e finalmente o tratamento Controle (CCC), em que os frangos não foram submetidos ao teste do halotano e nem ao estresse térmico. $\mathrm{O}$ pH inicial do músculo Pectoralis major $m$ e o valor de $\mathrm{R}$ foram medidos aproximadamente 30 min post mortem e as análises de $\mathrm{pH}$ final, cor $\left(\mathrm{L}^{*}, \mathrm{a}^{*}, \mathrm{~b}^{*}\right)$, Capacidade de Retenção de Água (CRA) foram realizadas $24 \mathrm{~h}$ após o abate nos filés mantidos a $4^{\circ} \mathrm{C}$. $\mathrm{O}$ valor de $\mathrm{pH}_{24 \mathrm{~h}}$ foi maior $(\mathrm{p} \leq 0,05)$ para os filés do CCC em comparação aos demais tratamentos e o valor de R foi maior para as aves HET e EET em comparação ao CCC $(\mathrm{p} \leq 0,05)$ sugerindo que o rigor mortis foi instalado mais rapidamente nas amostras derivadas das aves submetidas ao estresse térmico. Os tratamentos HHH, HET e EET apresentaram 4 aves cada com carnes PSE e 2 aves do tratamento CCC originaram filés PSE, demonstrando uma interferência tanto do halotano quanto do estresse térmico sobre o bem-estar das aves. Assim, os resultados demonstram que o halotano mostrou ser um agente estressor desencadeando alterações bioquímicas nos músculos das aves semelhantes às desencadeadas pelo estresse térmico pré-abate.

Palavras-chave: Bem-estar animal, qualidade da carne, carnes PSE

\begin{abstract}
Studies have been demonstrating in poultry, the existence of at least two causes for the formation of PSE meat: genetic through the sensibility towards halothane and the environmental factor in particular the temperature as the main factor. Thus the objective of this work was to evaluate halothane as stressor agent for broiler concomitantly to evaluating the PSE incidence by submitting these birds to thermal stress. In this experiment, 24 broilers of commercial lineage were divided in 6 animals for 4 treatments: $\mathrm{HHH}$, broilers submitted to halothane test and slaughtered $1 \mathrm{~h}$ after this test; HET broilers submitted to halothane at $35^{\circ} \mathrm{C} / 1 \mathrm{~h}$ after $48 \mathrm{~h}$ of halothane test followed by the birds slaughtering; EET, broilers
\end{abstract}

\footnotetext{
1 Químico, Doutorando do Programa de Pós-graduação em Ciência e Alimentos / CCA / Universidade Estadual de Londrina. E-mail: dfmarchi@yahoo.com.br

2 Professor Dr do Departamento de Zootecnia / CCA/ Universidade Estadual de Londrina. E-mail: oba@uel.br

3 Química / CCE / Universidade Estadual de Londrina. E-mail: gleice_quimica@hotmail.com

4 Professora Dra do Departamento e Ciência e Tecnologia de Alimentos / CCA/ Universidade Estadual de Londrina. E-mail: adri. soares@uel.br

5 Professor Dr do Departamento de Ciência e Tecnologia de Alimentos / CCA / Universidade Estadual de Londrina. Campus Universitário, Caixa Postal 6001, CEP 86081-990, Londrina-Pr, Brasil, fone (43) 3371-4984. E-mail: mshimo@uel.br

* Autor para correspondência
} 
submitted to thermal stress and slaughtered immediately after this treatment, and finally the control treatment (CCC) where broilers were not submitted to halothane test nor to thermal stress as control. The initial $\mathrm{pH}$ of Pectoralis major $\mathrm{m}$, was evaluated $30 \mathrm{~min}$ post mortem and the $\mathrm{pH}$ final, color, (L*, $\mathrm{a}^{*}$, $\mathrm{b}^{*}$ ) water holding capacity (WHC), and R value analysis were carried out after $24 \mathrm{~h}$ of storage of fillet samples at $4^{0} \mathrm{C}$. The $\mathrm{pH}_{24 \mathrm{~h}}$ was higher $(\mathrm{p} \leq 0.05)$ for $\mathrm{CCC}$ samples in comparison to other treatments and $\mathrm{R}$ value was higher for birds under HET and EET treatments in comparison to CCC $(\mathrm{p} \leq 0.05)$ suggesting that the rigor mortis was more rapid in samples from birds submitted to thermal stress. The HHH, HET, and EET treatments presented 4 birds each with PSE meat and 2 broilers from CCC treatment originated PSE meat showing the influence not only halothane but also the thermal stress over broilers welfare. Finally, results demonstrated that halothane is a stressor agent as well as the thermal stress and both promoted dramatic biochemical changes bringing about the formation of broiler PSE meat.

Key words: Animal welfare, meat quality, PSE meat

\section{Introdução}

A produção animal é o resultado do potencial genético das espécies que pode ser afetado por diversos fatores, como nutrição, sanidade, manejo e fatores ambientais, sendo que muitos animais não conseguem expressar todo o seu potencial produtivo em decorrência de condições adversas do meio em que vivem. Assim, o ambiente constitui um dos responsáveis pelo sucesso ou fracasso dos empreendimentos, uma vez que pode ser definido como a soma dos impactos dos meios biológicos e físicos circundantes sobre os animais (CURTIS, 1983).

Dentre os fatores ambientais, os fatores térmicos representados por temperatura, umidade do ar, radiação térmica e movimentação do ar são os principais agentes estressores em aves de corte. Animais submetidos a condições estressantes têm seu metabolismo acelerado, devido à liberação desordenada de cálcio, levando a um aumento na temperatura corporal (LESIÓW; KIJOWSKI, 2003). Assim, para manterem a temperatura interna do corpo em níveis relativamente constantes, ocorrem variações bioquímicas e fisiológicas para a manutenção da homeostase (BAETA; SOUZA, 1998).

Como conseqüência dessas alterações metabólicas há um aumento da produção de ácido láctico e da rigidez muscular (LESIÓW; KIJOWSKI, 2003; OWENS et al, 2000a). O rápido aumento da acidez muscular, associado à alta temperatura corporal, logo após o abate, causa a desnaturação de proteínas, ocasionando a diminuição da capacidade de retenção de água (CRA) e palidez do músculo (MCKEE; SAMS, 1997; VAN LAAK et al., 2000), os quais influenciam negativamente na qualidade da carne e caracteriza as carnes PSE (Pale, Soft, Exudative) (SAMS, 1999).

Estudos realizados com suínos mostram uma relação entre carnes PSE e a Síndrome do Estresse Suíno (PSS - Porcine Stress Syndrome) relacionada com a excessiva liberação de íons $\mathrm{Ca}^{2+}$ celular durante a contração muscular (MITCHELL; HEFFRON, 1982; BERTOL, 2005). Essa relação foi comprovada por Fujii et al. (1991) que detectaram um ponto de mutação no gene ryr1, responsável por codificar a proteína denominada receptora de rianodina do tipo 1 (RyR1) ou canal liberador de íons $\mathrm{Ca}^{2+}$ localizado no retículo sarcoplasmático do músculo esquelético (O’BRIEN, 1995).

A PSS foi inicialmente detectada com auxílio do halotano, um anestésico capaz de promover a rigidez muscular dos membros de suínos portadores dessa síndrome (BERTOL, 2005). A ação do halotano também foi avaliada em perus, em que foi constatado que os mesmos apresentavam reação similar à encontrada em suínos (WHEELER et al., 1999; OWENS, 2000a; OWENS et al., 2000b). Em relação à ação do halotano em frangos, o que se sabe até o momento é que este anestésico desencadeia reações que levam ao enrijecimento dos membros inferiores destes animais, porém sem 
nada conclusivo quanto ao seu efeito como agente estressor e sua avaliação na ocorrência de carnes PSE (MARCHI et al., 2008).

Assim, pesquisas que possam elucidar a ação dos principais agentes estressores em frangos através de parâmetros bioquímicos e fisiológicos, são de suma importância para uma descrição objetiva da anomalia PSE. Portanto, o objetivo do trabalho foi avaliar o halotano como agente estressor em frangos, tomando-se como parâmetro o estresse térmico.

\section{Material e Métodos}

\section{Animais}

Os frangos $(n=24)$ de linhagem comercial foram criados na Fazenda Escola da Universidade Estadual de Londrina - UEL até a realização do experimento. Os animais foram separados em 4 tratamentos, sendo 6 frangos por tratamento, conforme segue abaixo:
Tratamento HHH: Frangos submetidos ao teste do halotano e abatidos após $1 \mathrm{~h}$ de aplicação do teste.

Tratamento HET: Frangos submetidos ao teste do halotano, estresse térmico $48 \mathrm{~h}$ após aplicação do teste e abatidos em seguida

Tratamento EET: Frangos submetidos ao estresse térmico em uma câmara térmica e posterior abate dos animais.

Tratamento CCC (Controle): Frangos não foram submetidos ao teste do halotano e nem ao estresse térmico.

O teste do halotano foi aplicado com o auxílio de uma câmara elaborada exclusivamente para este experimento. A mesma apresenta três entradas frontais, onde foi inserida a cabeça de cada frango, e dimensões internas de $12 \times 12 \times 100 \mathrm{~cm}$. A câmara foi interligada a um aparelho de anestesia veterinária acoplado a um cilindro de $\mathrm{O}_{2}$ conforme ilustrado na (Figura 1).

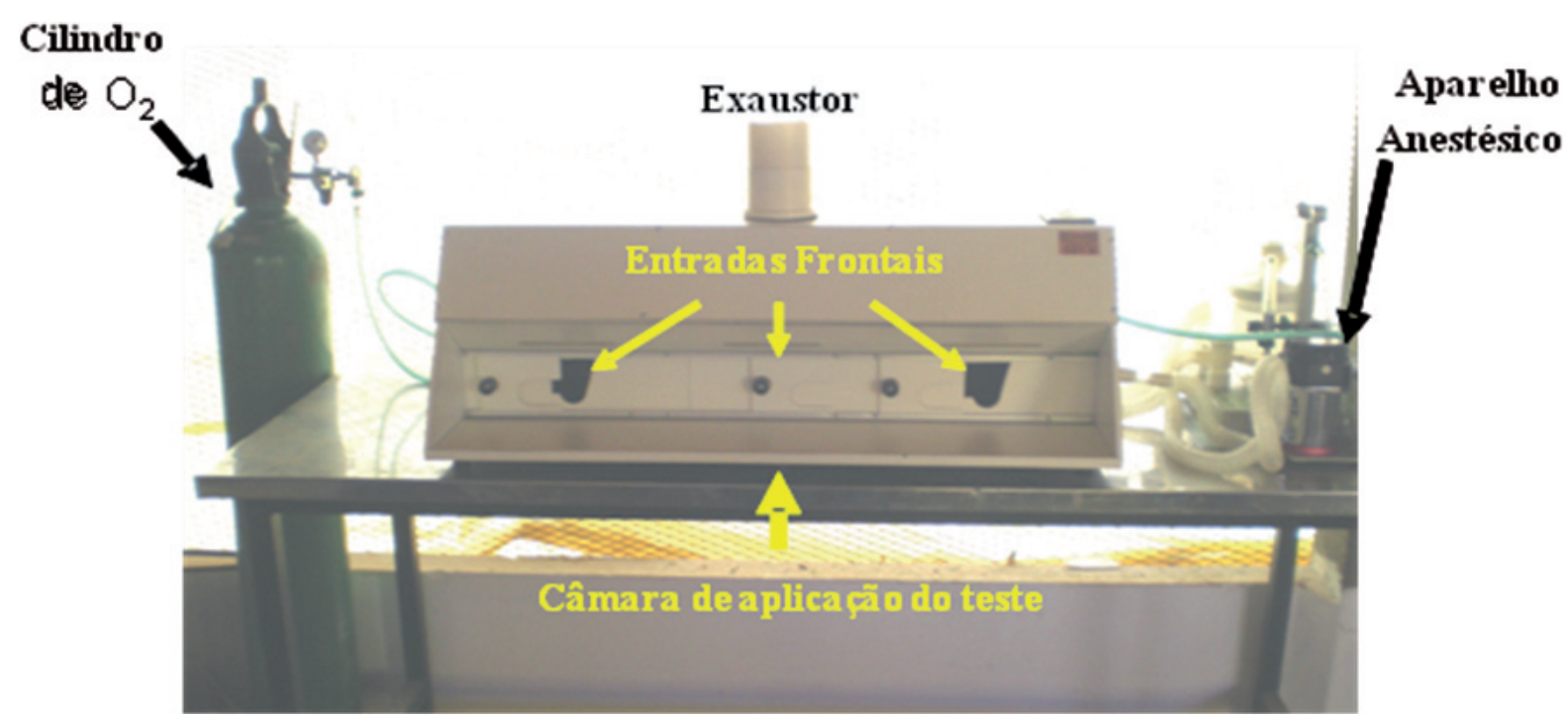

Figura 1. Câmara de aplicação do teste do halotano (MARCHI et al., 2008). 
Assim, o oxigênio foi liberado do cilindro com vazão de $6 \mathrm{~L} / \mathrm{min}$. chegando ao aparelho de anestesia veterinária, onde foi mesclado com o halotano a uma concentração de 3,0\%, e posteriormente, enviado para a câmara e inalado pelos frangos por $5 \mathrm{~min}$ (WHEELER et al., 1999, OWENS, 2000a; OWENS et al., 2000b; MARCHI et al., 2008).

O estresse térmico foi aplicado com auxílio de uma câmara térmica com dimensões internas próximas de 80x80x80cm, entrada de ar e um termostato acoplado, o qual foi regulado a $35^{\circ} \mathrm{C}$. Os frangos, em numero de 6 animais por vez foram deixados na câmara térmica onde permaneceram por $1 \mathrm{~h}$, imediatamente antes do abate.

As aves do grupo controle e tratamento $\mathrm{HHH}$ foram abatidas com 44 dias de idade. Os frangos dos tratamentos HET e ET foram abatidos com 46 dias de idade. $\mathrm{O}$ abate foi realizado de acordo com os procedimentos de abate de uma planta comercial, isto é, com insensibilização, sangria, escaldagem, depenagem, evisceração, chiller, desossa e refrigeração (NORTHCUTT, 2001; GUARNIERI; SOARES; SHIMOKOMAKI, 2004). Neste experimento, as aves não foram deixadas em jejum.

\section{Análises no músculo Pectoralis major}

As medidas de $\mathrm{pH}$ inicial e $\mathrm{pH}$ final foram realizadas aproximadamente $30 \mathrm{~min}$ e $24 \mathrm{~h}$ post mortem, respectivamente, diretamente no filé com auxílio de um potenciômetro de contato (Testo 205). Os pontos de incisão do eletrodo foram na parte cranial ventral do filé conforme descrito por Boulianne e King (1995) e adaptado por Olivo et al. (2001).Amedida de cor foi realizada $24 \mathrm{~h}$ post mortem em três pontos distintos de cada filé com o auxílio de um colorímetro Minolta CR400, iluminante D65 com ângulo de visão de $10^{\circ}$. Os valores medidos foram $\mathrm{L}^{*}$ (luminosidade), a* (componente verdevermelho) $b^{*}$ (componente azul-amarelo), expressos pelo sistema de cor CIELab.

A avaliação da CRA foi realizada $24 \mathrm{~h}$ post mortem conforme procedimento descrito por Hamm
(1960). A amostra utilizada para esta análise foi coletada na parte cranial do filé. O procedimento foi realizado em duplicata, em que a amostra foi coletada em cubo com um peso inicial de 2,0 g. Em seguida, a amostra foi colocada entre dois papéis filtro e posteriormente entre duas placas de acrílico. Este sistema foi deixado sob um peso de $10 \mathrm{~kg}$ por 5 min. Finalmente, a carne foi pesada e o cálculo da média de CRA determinado utilizando a fórmula abaixo:

$$
\left.\mathrm{CRA}=100-\frac{\left(\mathrm{P}_{\text {inicial }}-\mathrm{P}_{\text {final }}\right.}{\left(\mathrm{P}_{\text {inicial }}\right)} \times 100\right)
$$

Em que $\mathrm{P}_{\text {inicial }}$ e $\mathrm{P}_{\text {final }}$ são os pesos iniciais e finais da carne, respectivamente.

\section{Análise do valor de $R$}

A determinação do valor de $\mathrm{R}$ é denominada como a relação entre inosina monofosfato (IMP) e adenosina trifosfato (ATP). Valores de R superiores a 1,10 demonstram a instalação de rigor mortis acelerado relacionado com a ocorrência de carnes PSE (HONIKEL, 1981). A determinação do valor de $\mathrm{R}$ foi realizada, em duplicata, em amostras de filés de frango coletadas da parte cranial do músculo Pectoralis major aproximadamente $30 \mathrm{~min}$ post mortem. As amostras permaneceram congeladas em nitrogênio líquido $\left(-196{ }^{\circ} \mathrm{C}\right)$ até realização das análises. A metodologia seguiu a técnica descrita de Honikel e Fischer (1977) em que a extração dos nucleotídeos em 2,0 g de amostra de filé foi obtida através de homogeneização por 30 seg em solução de ácido perclórico $1 \mathrm{M}$, na proporção de 1:10 em p/v. A seguir, esta foi filtrada e centrifugada por $5 \mathrm{~min}$ a $4800 \mathrm{rpm}$. Em seguida, uma alíquota de 0,2 $\mathrm{mL}$ do sobrenadante foi diluída em $4,8 \mathrm{~mL}$ de tampão fosfato a 0,1 M em pH 7,0 e lida em espectrofotômetro a 250 $\mathrm{nm}$ (monofosfato de inosina) e $260 \mathrm{~nm}$ (trifosfato de adenosina). O tampão fosfato foi utilizado como branco e o valor de $\mathrm{R}$ foi calculado como a razão entre as duas absorbâncias. 


\section{Classificação de Carnes PSE}

A classificação de carnes PSE foi realizada, tomando-se o valor de $\mathrm{L}^{*}$ como parâmetro determinante, em que filés com valores de $\mathrm{L}^{*} \geq$ 53,0 foram classificados como PSE, conforme padronizado por Soares et al. (2002).

\section{Análise Estatística}

O programa STATISTICA for Windows versão 6.0 foi utilizado para a análise dos resultados. $\mathrm{O}$ teste de média de Tukey a $5 \%$ de probabilidade foi aplicado para comparar as diferenças significativas entre os 4 tratamentos em relação às medidas de $\mathrm{pH}$ inicial e final, Cor ( $\left.\mathrm{L}^{*}, \mathrm{a}^{*}, \mathrm{~b}^{*}\right), \mathrm{CRA}$, valor de R.

\section{Resultados e Discussão}

Neste experimento, todas as aves avaliadas pelo teste do halotano saíram desacordadas da câmara de aplicação do teste, e permaneceram neste estado por aproximadamente 2 a 3 min demonstrando a ação anestésica do mesmo, o que está de acordo com Bennett (1992), que relataram que a recuperação anestésica do halotano pode variar de 3 a $5 \mathrm{~min}$.

$\mathrm{Na}$ Tabela 1 estão apresentados os valores das medidas de $\mathrm{pH}$ inicial e final, Cor e CRA dos filés de peito de frango submetidos aos diferentes tratamentos.

Tabela 1. Valores médios de $\mathrm{pH}$ inicial $\left(\mathrm{pH}_{\mathrm{i}}\right)$ e final $\left(\mathrm{pH}_{\mathrm{f}}\right)$, Cor $\left(\mathrm{L}^{*}, \mathrm{a}^{*}, \mathrm{~b}^{*}\right)$ e Capacidade de Retenção de Água (CRA) dos filés de peito de frangos submetidos aos tratamentos: HHH (submetidos ao teste do halotano), HET (submetidos ao teste do halotano e ao estresse térmico por $35^{\circ} \mathrm{C} / 1 \mathrm{~h}$ após $48 \mathrm{~h}$ do teste halotano), EET (apenas submetidos ao estresse térmico de $35^{\circ} \mathrm{C} / 1 \mathrm{~h}$ ) e CCC (controle, sem teste do halotano e sem estresse térmico).

\begin{tabular}{ccccccc}
\hline Tratamentos & $\mathbf{p H}_{\mathbf{i}}$ & $\mathbf{p H}_{\mathbf{f}}$ & $\mathbf{L}^{*}$ & $\mathbf{a}^{*}$ & $\mathbf{b}^{*}$ & $\mathbf{C R A}(\mathbf{\%})$ \\
\hline \multirow{2}{*}{ HHH } & 6,09 & $5,79^{\mathbf{b}}$ & 53,92 & 3,59 & 7,37 & 70,51 \\
& $\pm 0,25$ & $\pm 0,10$ & $\pm 2,57$ & $\pm 1,40$ & $\pm 0,75$ & $\pm 3,91$ \\
\multirow{2}{*}{ HET } & 6,31 & $5,79^{\mathbf{b}}$ & 53,61 & 3,26 & 7,46 & 72,76 \\
& $\pm 0,23$ & $\pm 0,08$ & $\pm 0,90$ & $\pm 1,35$ & $\pm 0,95$ & $\pm 1,70$ \\
& 6,11 & $5,76^{\mathbf{b}}$ & 53,92 & 2,84 & 7,29 & 70,51 \\
EET & $\pm 0,16$ & $\pm 0,05$ & $\pm 2,57$ & $\pm 0,91$ & $\pm 1,14$ & $\pm 3,91$ \\
& 6,25 & $5,92^{\mathbf{a}}$ & 52,00 & 3,01 & 7,08 & 72,78 \\
& $\pm 0,25$ & $\pm 0,09$ & $\pm 2,78$ & $\pm 0,84$ & $\pm 0,79$ & $\pm 2,08$ \\
\hline
\end{tabular}

Médias seguidas de letras diferentes na mesma coluna diferem entre si pelo teste de Tukey a 5,0\% de probabilidade ( $\mathrm{p} \leq 0,05)$.

\pm Desvio-padrão

Dos parâmetros avaliados, apenas o $\mathrm{pH}$ final diferiu significativamente $(p<0,05)$ entre $o$ controle e os demais tratamentos. O menor $\mathrm{pH}$ observado nos filés de frango dos tratamentos HHH, HET e EET está relacionado com o estresse destes animais, responsável pela maior produção de ácido lático durante o rigor mortis. Observa-se também que os valores de $L^{*}$, referentes a estes três grupos, foram superiores a 53, demonstrando que as aves destes tratamentos apresentaram seus filés caracterizados como PSE, relacionando-se ao $\mathrm{pH}$, ou seja, quanto menor o $\mathrm{pH}$ maior a luminosidade, e mais pálido apresenta-se o filé (OLIVO et al., 2001; ODA, 2006).

\section{Avaliação do Valor de $R$}

A medida do valor de $\mathrm{R}$ para os tratamentos $\mathrm{HHH}$, HET, EET e CCC está apresentada na (Tabela 2). 
Tabela 2. Valor de R de filés de frango dos tratamentos: HHH (submetidos ao teste do halotano), HET (submetidos ao teste do halotano e ao estresse térmico de $35^{\circ} \mathrm{C} / 1 \mathrm{~h}, 48 \mathrm{~h}$ após o teste halotano), EET (submetidos ao estresse térmico a $\left.35^{\circ} \mathrm{C} / 1 \mathrm{~h}\right)$ e Controle.

\begin{tabular}{ccc}
\hline Tratamentos & Número de filés de frango analisados & Valor de R \\
\hline HHH & 2 & $1,15^{\mathrm{ab}} \pm 0,06$ \\
HET & 4 & $1,18^{\mathbf{a}} \pm 0,03$ \\
EET & 4 & $1,22^{\mathrm{a}} \pm 0,02$ \\
CCC & 4 & $1,11^{\mathbf{b}} \pm 0,02$ \\
\hline
\end{tabular}

Médias seguidas de letras diferentes na mesma coluna diferem entre si pelo teste Tukey 5,0\% de probabilidade $(\mathrm{p} \leq 0,05)$.

\pm Desvio-padrão

Embora os valores de R sejam todos superiores a 1,10 foi possível evidenciar diferença significativa $(\mathrm{p} \leq 0,05)$ entre os filés do tratamento controle e os demais. As aves dos tratamentos que sofreram ação do estresse térmico (HET e EET) apresentaram maiores valores $(\mathrm{p} \leq 0,05)$ do que o grupo controle demonstrando que os frangos destes tratamentos apresentaram estágios mais elevados de rigor mortis, com produção mais acelerada de IMP e, conseqüentemente apresentaram menores concentrações de ATP (HONIKEL; FISCHER, 1977).

Estes resultados foram semelhantes aos relatados por Bressan e Beraquet (2002), que afirmaram que as aves submetidas, no pré-abate, ao excesso de calor apresentam aceleração da glicólise e hidrólise de ATP. Entretanto, os mesmos autores observaram que em temperaturas ambientais baixas essa evolução foi retardada. Resultados similares também foram encontrados por Bressan e Beraquet (2002), em que o tratamento com temperatura em torno de $30^{\circ} \mathrm{C}$ foi suficiente para promover maior valor de $\mathrm{R} \mathrm{em}$ filés quando comparados aos peitos dos frangos submetidos à temperatura de $17^{\circ} \mathrm{C}$.

Por outro lado, os filés do tratamento $\mathrm{HHH}$ apresentaram valores de $\mathrm{R}$ similares aos demais tratamentos, indicando que o halotano não influenciou tanto quanto o estresse térmico na hidrólise do ATP.
Incidência de Carnes com Características PSE sob Influência do Estresse Térmico e do Halotano

A ocorrência de carnes caracterizadas como PSE nos tratamentos HHH, HET, EET e Controle está apresentada na (Figura 2).

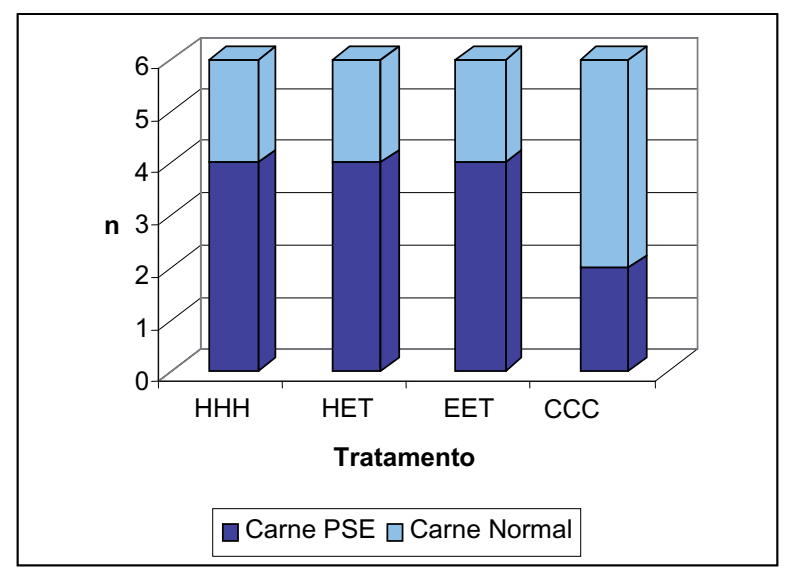

Figura 2. Ocorrência de carnes com características PSE em frangos dos tratamentos $\mathrm{HHH}$ (submetidos ao teste do halotano), HET (submetidos ao teste do halotano e ao estresse térmico a $35^{\circ} \mathrm{C} / 1 \mathrm{~h}$ e $48 \mathrm{~h}$ após o teste halotano), EET (submetidos ao estresse térmico a $35^{\circ} \mathrm{C} / 1 \mathrm{~h}$ ) e Controle.

Os resultados indicam que o halotano apresenta influência como agente estressor similar, ao encontrado em aves submetidas ao estresse térmico, o que pode ser constatado pelo maior número de filés PSE, quando comparados com o tratamento Controle. O efeito do estresse térmico sobre o desenvolvimento de carnes PSE já vêm sendo descrito por vários pesquisadores (OLIVO et al., 2001; BRESSAN; 
BERAQUET, 2002; SOARES et al., 2003; AKSIT et al., 2006). Entretanto, alguns autores relatam que o estresse térmico por si só não é capaz de determinar a ocorrência significativa de carnes PSE, sugerindo que esta anomalia é conseqüência de uma interação de fatores ambientais e genéticos, onde um único fator tomado isoladamente não pode caracterizar satisfatoriamente esta síndrome (LARA; SHIMOKOMAKI, 2002).

Entretanto, o fato de o tratamento Controle ter apresentado carnes com as características PSE, embora em quantidade menor, demonstra que fatores pré-abate podem influenciar na qualidade final da carne.

\section{Conclusão}

$\mathrm{O}$ anestésico halotano mostrou ser um agente estressor desencadeando alterações bioquímicas nos músculos dos frangos semelhantes às desencadeadas pelo estresse térmico pré-abate.

\section{Referências}

AKSIT, M. Effects of temperature during rearing and crating on stress parameters and meat quality of broilers. Poultry Science, Champaing, v. 85, n. 11, p. 1867-1874, 2006.

BAETA, F. C.; SOUZA, C. F. Ambiência em edificações rurais- conforto animal. Viçosa: UFV. 1998. 246 p.

BENNETT, R. A. Current methods in avian anesthesia. In: ACVS VETERINARY SYMPOSIUM, 20, 1992, Miami. Proceedings... Miami: American College of Veterinary Surgeons, 1992. p. 636.

BERTOL, T. M. Effects of dietary supplementation with L-carnitine and fat on blood acid-base responses to handling in slaughter weight pigs. Journal Animal Science, New York, v. 83, n. 3, p. 75-81, 2005.

BOULIANNE, M; KING, A. J. Biochemical and color characteristics of skinless boneless pale chicken breast. Poultry Science, Champaign, v. 74, n. 11, p. 1693-1698, 1995.
BRESSAN, M. C.; BERAQUET, N. J. Efeito de fatores pré-abate sobre a qualidade da carne de peito de frango. Revista de Ciência e Agrotecnologia, Lavras, v. 26, n. 5, p. 1049-1059, 2002.

BRESSAN, M. C. Como diminuir o estresse causado pela apanha, transporte e abate visando o bem-estar de frangos. In: CONFERÊNCIA APINCO DE CIÊNCIA E TECNOLOGIA AVÍCOLAS, 2003, Campinas. Anais... Campinas: FACTA, p. 255-268, 2003.

CURTIS, S. E. Environmental manegemente in animal agriculture. 2. ed. Ames, Towa: Iwoa State University. 1983. 407 p.

FUJII, J.; OTSU, K.; ZORZATO, F.; LEON, S.; KHANNA, V. K.; WEILER, J. E; O`BRIEN, P. J.; MacLENNAN, D. H. Identification of mutation in porcine ryanodine receptor associated with malignant hyperthermia. Science, Washington, v. 253, n. 3, p. 448451, 1991

GUARNIERI, P. D.; SOARES, A. L.; OLIVO, R.; SCHNEIDER, J. P.; MACEDO, R. M.; IDA, E. I.; SHIMOKOMAKI, M. Preslaughter handling with water shower spray inhibits PSE (Pale, Soft, Exudative) broiler breast meat in a commercial plant. Biochemical and ultrastructural observations. Journal of Food Biochemistry, Trumbull, v. 28, n. 4, p. 269-277, 2004.

HAMM, R. Biochemistry of meat hydratation. Advances in Food Research, Cleveland, v. 10, n. 2, p. 335-443, 1960.

HONIKEL, K. O. Influence of post mortem changes in bovine muscle on water-holding capacity of beef. Post mortem storage of muscle at $20^{\circ} \mathrm{C}$. Journal of Food Science, Chicago, v. 46, n. 1, p. 1-7, 1981.

HONIKEL, K. O.; FISCHER, C. A. A rapid method for the detection of PSE and DFD porcine muscle. Journal of Food Science, Chicago, v. 42, n. 7, p. 1663-1676, 1977.

LARA, J. A. F.; SHIMOKOMAKI, M. Estresse térmico e carne PSE em frangos. In: CONFERÊNCIA APINCO DE CIÊNCIA E TECNOLOGIA AVÍCOLA, 2002, Campinas. Anais... Campinas: FACTA, v. 3. p. 19-19, 2002.

LESIÓW, T.; KIJOWSKI, J. Impact of PSE and DFD meat on poultry processing. A review. Polish Journal of Food and Nutrition Sciences, Olsztyn, v. 12/53, n. 2, p. 3-8, 2003.

MARCHI, D. F.; OBA, A.; SOARES, A. L.; IDA, E. I.; SHIMOKOMAKI, M. Genetic origin of Broiler PSE Meat. Brisbane, Australia: World's Poultry Congress, 2008. 
McKEE, S. R.; SAMS, A. R. The effect of seasonal heat stress on rigor development and the incidence of pale, exsudative turkey meat. Poultry Science, Champaign, v. 76, n. 11, p. 1616-1620, 1997.

MITCHELl, G.; HEFFRON, J. J. A. Porcine stress syndromes. In: CHICHESTER, C.O. (Ed.). Advances in food research. New York: Academic Press, 1982. v. 28. p. 167-230.

NORTHCUTT, J. K. Preslaughter factors affecting poultry meat quality. In: SAMS, A. R. (Ed.). Poultry meat processing. Boca Raton: CRC Press, 2001. p. 5-18.

O'BRIEN, P. J. The causative mutation for porcine stress syndrome. Food Animal, New York, v. 17, n. 2, p. $257-$ 269, 1995.

ODA, S. H. I. Análises moleculares do gene codificador da proteina receptora de rianodina e a ocorrência de carnes PSE (Pale, Soft, Exudative) em frangos. 2006. Tese. (Doutorado em Ciência de Alimentos) Departamento de Ciência e Tecnologia de Alimentos. Universidade Estadual de Londrina, Londrina.

OLIVO,R.;SOARES,A.L.;IDA,E.I.;SHIMOKOMAKI, M. Dietary vitamin E inhibits poultry PSE and improves meat functional properties. Journal of Food Biochemistry, Trumbull, v. 25, n. 4, p. 271-283, 2001.
OWENS, C. M. The development of pale, exudative meat in two genetic lines of turkeys subjected to heat stress and its prediction by halothane screening. Poultry Science, Champaign, v. 79, n. 3, p. 430-435, 2000a.

OWENS, C. M.; MATHEWS, N. S.; SAMS, A. R. The use of halothane gas to identify turkeys prone to pale, exudative meat when transported before slaughter. Poultry Science, Champaing, v. 79, n. 5, p. 789-795, 2000b.

SAMS, A. R. Meat quality during processing. Poultry Science, Champaign, v. 78, n. 4, p. 798-803, 1999.

SOARES, A. L.; LARA, J.A. F.; IDA, E. I.; GUARNIERI, P. D.; OLIVO, R.; SHIMOKOMAKI, M. Variation in the color of Brazilian broiler breast fillet. Proceedings of International Congress of Meat Science and Technology, Roma, v. 48, p. 540-541, 2002.

VAN LAACK, R. L.; LUI, C. H.; SMITH, M. O.; LOVEDAY, H. D. Characteristics of pale soft, exudative broiler breast meat. Poultry Science, Champaing, v. 79, n. 7, p. 1057-1061, 2000.

WHEELER, B. R.; McKEE, S. R.; MATTHEWS, N. S.; MILLER, R. K.; SAMS, A. R. A halothane test to detect turkeys prone to developing pale, soft, and exudative meat. Poultry Science, Champaing, v. 78, n. 11, p. 16341638, 1999. 